\title{
On parametric solution of fault detection problems
}

\author{
Andras Varga \\ German Aerospace Center, DLR-Oberpfaffenhofen \\ Institute of Robotics and Mechatronics, D-82234 Wessling, Germany \\ (e-mail: andras.varga@dlr.de)
}

\begin{abstract}
We discuss the solution of the fault detection problem in presence of parametric uncertainties. The basic approach is an extension of the nullspace method for constant systems to the case of linear parameter varying (LPV) models. In a general setting, we consider the case when part of the unknown parameters are non-measurable and part of them are measurable. The resulting LPV-gain scheduled fault detection filter provides robustness with respect to both types of parametric uncertainties. Symbolic and numerical computational procedures which underlie the proposed synthesis approach are discussed.
\end{abstract}

Keywords: Fault detection, LPV systems, numerical algorithms, symbolic methods.

\section{INTRODUCTION}

Addressing robustness aspects in the synthesis of residual generators is a active area of present day fault detection research. For the synthesis of robust residual generators several techniques have been proposed. A popular approach, mainly advocated in (Chen and Patton, 1999), is to recast uncertain parameters as fictive inputs and synthesize robust residual generators using exact or approximate synthesis techniques. The difficulties with this technique lie in the need to produce meaningful models which can be used for synthesis and in the need of an $a$ posteriori robustness analysis. Also, this method is not appropriate for a gain-scheduling based approach, provided some of the uncertain parameters are measurable.

Linear parametric varying (LPV) models can be used to approximate nonlinear models or to represent linear models depending on several uncertain parameters. Such models can be obtained in several ways, see for example (Varga et al., 1998; Pfifer and Hecker, 2008). Geometric synthesis methods employing LPV-models with affine parameter dependence have been proposed in (Balas et al., 2003). The application of these methods to fault detection filter designs is described in (Bokor and Balas, 2004). The main appeal of using the LPV formalism is that the solutions can be obtained using linear algebraic manipulations like those elaborated for linear time-invariant (LTI) systems in (Wonham, 1979). This approach is primarily intended for robust fault detection using LPV-based gain scheduling schemes in the case when all uncertain parameters are measurable, and is less suited to address the robust synthesis of fault detection filters in the presence of parametric uncertainties.

In this paper we propose a general approach to address robust fault detection problems, where part of uncertain parameters is measurable and thus can be used for gain

* This work was performed in the framework of the ADDSAFE Project: Grant agreement no.: FP7-233815. scheduling, and part is nonmeasurable, for which robustness must be enforced. The proposed procedure extends the nullspace approach of Frisk and Nyberg (2001) and Varga (2008a) to LPV models and provides a way to achieve via a partial LPV-based gain scheduling robustness simultaneously with respect to measurable and nonmeasurable uncertain parameters. Symbolic linear algebra tools for rational nullspace computation and numerical robust optimization techniques for worst-case parameter fitting are required to perform the proposed synthesis approach. For both of them, adequate computational tools are available in standard computational environments like MATLAB and Maple. The robust fault detection and isolation can be achieved by using a bank of detectors providing a structured residual set. An example illustrates the potentials of the proposed method.

\section{THE ROBUST FAULT DETECTION PROBLEM}

Consider additive fault models described by state-space LPV representations of the form

$$
\begin{aligned}
& \dot{x}(t)=A(\rho) x(t)+B_{u}(\rho) u(t)+B_{d}(\rho) d(t)+B_{f}(\rho) f(t) \\
& y(t)=C(\rho) x(t)+D_{u}(\rho) u(t)+D_{d}(\rho) d(t)+D_{f}(\rho) f(t)
\end{aligned}
$$

where $x(t)$ is the $n$-dimensional system state vector, $y(t)$ is the $p$-dimensional system output vector, $u(t)$ is the $m_{u}$-dimensional control input vector, $d(t)$ is the $m_{d^{-}}$ dimensional disturbance vector, and $f(t)$ is the $m_{f^{-}}$ dimensional fault vector $f(t)$.

Throughout the paper we will assume that the system matrices $A(\rho), B_{u}(\rho), \ldots$ depend rationally of the components of the parameter vector $\rho$ and therefore their entries belong to the field $\mathrm{K}(\rho)$ of multivariate rational functions in the components of vector $\rho$. The vector $\rho$ is assumed to belong to a bounded region $\Pi \subset \mathcal{P}$ of the $n_{\rho}$-dimensional parameter space $\mathcal{P}$. The value of $\rho$ is generally assumed to be a priori unknown, but in some applications $\rho$ or a part of its components can be measured. In a more 
general setting we can also allow time-varying parameters $\rho(t)$. However, to simplify the notations, the dependence of time of $\rho$ will not be explicitly emphasized and only slowly varying parameters which can be assimilated with constant values over sufficiently large period are considered in our study.

To ease the presentation, we will assume that the parameters are constant. Thus, we can alternatively use an inputoutput representation of the form

$$
\mathbf{y}(s)=G_{u}(s, \rho) \mathbf{u}(s)+G_{d}(s, \rho) \mathbf{d}(s)+G_{f}(s, \rho) \mathbf{f}(s),
$$

where $\mathbf{y}(s), \mathbf{u}(s), \mathbf{d}(s)$, and $\mathbf{f}(s)$ are the Laplace-transformed vectors $y(t), u(t), d(t)$, and $f(t)$, respectively, and $G_{u}(s, \rho), G_{d}(s, \rho)$, and $G_{f}(s, \rho)$ are the parameter dependent transfer-function matrices (TFMs) from the control inputs to outputs, disturbance inputs to outputs, and fault inputs to outputs, respectively. These TFMs are given by

$$
\begin{aligned}
& G_{u}(s, \rho)=C(\rho)(s I-A(\rho))^{-1} B_{u}(\rho)+D_{u}(\rho) \\
& G_{d}(s, \rho)=C(\rho)(s I-A(\rho))^{-1} B_{d}(\rho)+D_{d}(\rho) \\
& G_{f}(s, \rho)=C(\rho)(s I-A(\rho))^{-1} B_{f}(\rho)+D_{f}(\rho)
\end{aligned}
$$

Regarding the unknown parameter vector $\rho$, generally we can assume that it has two components: $\rho_{1} \in \Pi_{1}$, which is not measurable, and $\rho_{2} \in \Pi_{2}$, which is measurable, and $\Pi=\Pi_{1} \times \Pi_{2}$. The synthesis problem formulated bellow attempts to solve basically a robust fault detection problem with respect to $\rho_{1}$, while taking advantage of the availability of $\rho_{2}$ by attempting to achieve robustness using an LPV gain scheduling approach.

A linear residual generator (or fault detection filter) processes the measurable system outputs $y(t)$ and control inputs $u(t)$ and generates the residual signals $r(t)$ which serve for decision making on the presence or absence of faults. We use a parameter dependent filter of the form

$$
\mathbf{r}(s)=Q\left(s, \rho_{2}\right)\left[\begin{array}{l}
\mathbf{y}(s) \\
\mathbf{u}(s)
\end{array}\right]
$$

where $Q\left(s, \rho_{2}\right)$ is the TFM of the filter, which explicitly depends on the measurable parameter $\rho_{2}$ (e.g., via an equivalent state-space realization of the filter). For a physically realizable filter, $Q\left(s, \rho_{2}\right)$ must be proper with respect to $s$ (i.e., only with finite poles) and robustly stable (i.e., only with poles having negative real parts for all values of $\rho_{2}$ ). The dimension $q$ of the residual vector $r(t)$ depends on the fault detection problem to be solved. The simpler case when only $\rho_{1}$ is present leads to a constant detector $Q(s)$, while in the absence of $\rho_{1}$ only the LPV gain scheduling aspect is addressed.

The residual signal $r(t)$ in (3) generally depends via the system outputs $y(t)$ of all system inputs $u(t), d(t)$ and $f(t)$. The residual generation system, obtained by replacing in (3) $\mathbf{y}(s)$ by its expression in (2), is given by

$$
\mathbf{r}(s)=R_{u}(s, \rho) \mathbf{u}(s)+R_{d}(s, \rho) \mathbf{d}(s)+R_{f}(s, \rho) \mathbf{f}(s)
$$

where

$$
\left[R_{u}(s, \rho)\left|R_{d}(s, \rho)\right| R_{f}(s, \rho)\right]:=Q\left(s, \rho_{2}\right) G_{e}(s, \rho)
$$

with

$$
G_{e}(s, \rho):=\left[\begin{array}{c|c|c}
G_{u}(s, \rho) & G_{d}(s, \rho) & G_{f}(s, \rho) \\
I_{m_{u}} & 0 & 0
\end{array}\right]
$$

For a successfully designed filter $Q\left(s, \rho_{2}\right)$, the corresponding residual generation system is proper with respect to variable $s$, robustly stable and achieves specific fault detection requirements (e.g., exact or approximate decoupling of control and disturbance inputs from the residuals).

We can now formulate the following Robust Fault Detection Problem (RFDP): For the LPV-system (2), determine a proper and stable linear residual generator (or fault detector) filter having the form (3) such that for all $\rho \in \Pi$ and a given $\gamma>0$ there exists $\beta>0$ such that

(i) $\quad\|r(t)\| \leq \gamma \max \{\|u(t)\|,\|d(t)\|\}$ when $f(t)=0$ and for all $u(t), d(t)$;

(ii) $\quad\|r(t)\| \geq \beta\left\|f_{i}\right\|$ for $i=1, \ldots, m_{f}$ and for $u(t)=0, d(t)=0$

(iii) $\quad r(t)$ is asymptotically bounded.

Here $\|\cdot\|$ denotes signal norms in appropriate Banach spaces (see for example (Zhou et al., 1996, page 92)). The gap defined as $\beta / \gamma$ measures the sensitivity of detection task, where larger values guarantees the detection of smaller faults. The exact solution of the RFDP corresponds to the case when we can choose $\gamma=0$, and the corresponding gap is $\infty$.

Besides the above requirements it is often desirable for practical use that the $q \times\left(p+m_{u}\right)$ TFM of the detector $Q\left(s, \rho_{2}\right)$ has the least possible McMillan degree. Note that as fault detector, we can always choose $Q\left(s, \rho_{2}\right)$ as a rational row vector, thus with $q=1$ (scalar) output. However, occasionally values $q>1$ could be more convenient to obtain a better conditioning of sensitivities of residuals to individual faults.

The fulfillment of requirement (ii) ensures that faults produce non-zero residual responses. When designing fault detectors, this requirement for fault detectability is usually replaced by the stronger condition that persistent (e.g., constant) faults produce asymptotically persistent residuals. This requirement is known as strong fault detectability.

The requirements $(i)$ and $(i i)$ can be easily transcribed into equivalent synthesis conditions. The approximate decoupling condition $(i)$ requires that both $R_{u}(s, \rho)$ and $R_{d}(s, \rho)$ have small norms $\forall \rho$, and thus can be achieved by minimizing the worst-case norm

$$
\max _{\rho \in \Pi}\left\|\left[R_{u}\left(s, \rho_{2}\right) R_{d}(s, \rho)\right]\right\|
$$

With a suitable scaling of the detector, we can always achieve that this worst-case norm is equal to a given $\gamma$.

The (detectability) condition $(i i)$ is equivalent to

$$
\beta:=\min _{i=1, \ldots, m_{f}} \min _{\rho \in \Pi}\left\|R_{f_{i}}(s, \rho)\right\|>0,
$$

where $R_{f_{i}}(s, \rho)$ is the $i$-th column of $R_{f}(s, \rho)$.

Additionally, the condition ( iii) on the boundedness of the residual signal requires that $R_{f}(s, \rho)$ as well as $R_{u}(s, \rho)$, and $R_{d}(s, \rho)$ are stable TFMs for all $\rho$. In the case when the $\rho_{1}$ component is present, a necessary condition to fulfill (iii) is the robust stabilizability of the plant (2) (Kinnaert et al., 1995). In what follows, we will tacitly assume that this condition is fulfilled.

In the next section we discuss how the solution of the formulated RFDP can be addressed using a combination of symbolic and numerical computational tools. 


\section{PARAMETRIC NULLSPACE METHOD}

\subsection{The basic approach}

Assume temporarily that $\rho$ is measurable. In this case, we can try to exactly solve the RFDP by determining stable and proper $Q(s, \rho)$ such that for all $\rho \in \Pi$

$$
Q(s, \rho) G(s, \rho)=0,
$$

where

$$
G(s, \rho)=\left[\begin{array}{cc}
G_{u}(s, \rho) & G_{d}(s, \rho) \\
I_{m_{u}} & 0
\end{array}\right]
$$

and

$$
R_{f_{i}}(s, \rho) \neq 0, \quad i=1, \ldots, m_{f} .
$$

In what follows, we assume that the standard solvability conditions (Nyberg, 2002) are fulfilled for all $\rho \in \Pi$ :

$\operatorname{rank}\left[G_{d}(s, \rho) G_{f_{i}}(s, \rho)\right]>\operatorname{rank} G_{d}(s, \rho), i=1, \ldots, m_{f}(11)$

From (8) it appears that $Q(s, \rho)$ is a left annihilator of $G(s, \rho)$, thus one possibility to determine $Q(s, \rho)$ is to compute first a parameter dependent minimal polynomial basis $N_{l}(s, \rho)$ for the left nullspace of $G(s, \rho)$, and then to build a stable detector as

$$
Q(s, \rho)=W(s) N_{l}(s, \rho),
$$

where $W(s)$ is a rational matrix with $q$ rows chosen such that conditions (10) jointly with the stability requirement on $R_{f}(s, \rho)$ are fulfilled. In general, $N_{l}(s, \rho)$ is a $\left(p-r_{d}\right) \times$ $\left(p+m_{u}\right)$ TFM, where $r_{d}:=\operatorname{rank} G_{d}(s, \rho)$. Due to practical considerations, the number of residual outputs $q$ is chosen such that $q \leq p-r_{d}$. Generally, smaller values of $q$ allow to determine detectors with smaller dynamical orders.

The expression (12) can be seen as a parametrization of all possible LPV fault detectors with $q$ outputs and is the basis of the so-called nullspace methods. For example, $W(s)$ can be chosen as $W(s)=h / d(s)$, where $h$ is a constant row vector and $d(s)$ is a polynomial chosen to make $h N_{l}(s, \rho) / d(s)$ proper and stable. Special choice of $h$ can ensure obtaining a detector of least McMillan degree (Varga, 2008b). Alternatively, $W(s)$ can be chosen as $W(s)=D^{-1}(s) H$, where $H$ is a constant matrix with $q$ rows and $D(s)$ is a $q \times q$ invertible polynomial matrix, chosen to ensure properness and stability of $D^{-1}(s) H N_{l}(s, \rho)$.

When $\rho$ contains a non-measurable component $\rho_{1}$, a detector of the form $Q(s, \rho)$ can not be implemented. However, $Q(s, \rho)$ can serve to obtain an approximation $\bar{Q}\left(s, \rho_{2}\right)$ by minimizing the worst-case error norm

$$
\max _{\rho \in \Pi}\left\|Q(s, \rho)-\bar{Q}\left(s, \rho_{2}\right)\right\|
$$

such that conditions (7) for $Q(s, \rho)=\bar{Q}\left(s, \rho_{2}\right)$ jointly with the stability requirement on $R_{u}(s, \rho), R_{d}(s, \rho)$ and $R_{f}(s, \rho)$ are fulfilled. Possible norms to be employed in (13) are the $\mathcal{H}_{2^{-}}, \mathcal{H}_{\infty^{-}}$, or even the $\nu$-gap norm (Vinnicombe, 1993). Alternatively, the weighted worst-case error norm

$$
\max _{\rho \in \Pi}\left\|\left(Q(s, \rho)-\bar{Q}\left(s, \rho_{2}\right)\right) G_{e}(s, \rho)\right\|
$$

can be minimized to cover both conditions (6) and (7).

\subsection{Computation of nullspace}

The computational method described in (Varga, 2003) for constant systems can be extended to LPV systems by exploiting the simple fact that $N_{l}(s, \rho)$ is a left nullspace basis of $G(s, \rho)$ iff $\left[M_{l}(s, \rho) N_{l}(s, \rho)\right]$ is a left nullspace basis of the system matrix

$$
S(s, \rho)=\left[\begin{array}{ccc}
A(\rho)-s I_{n} & B_{u}(\rho) & B_{d}(\rho) \\
C(\rho) & D_{u}(\rho) & D_{d}(\rho) \\
0 & I_{m_{u}} & 0
\end{array}\right]
$$

Thus, to compute $N_{l}(s, \rho)$ we can determine equivalently a left nullspace basis $Y_{l}(s, \rho)$ of $S(s, \rho)$ and then $N_{l}(s, \rho)$ simply results as

$$
N_{l}(s, \rho)=Y_{l}(s, \rho)\left[\begin{array}{c}
0 \\
I_{m_{u}+p}
\end{array}\right] .
$$

Moreover, it can be shown that

$$
N_{l}(s, \rho)\left[\begin{array}{c}
G_{f}(s, \rho) \\
0
\end{array}\right]=Y_{l}(s, \rho)\left[\begin{array}{c}
B_{f}(\rho) \\
D_{f}(\rho) \\
0
\end{array}\right]
$$

which allows to easily check the solvability condition (11), by checking instead (7) for $Q(s, \rho)=N_{l}(s, \rho)$.

The matrix $S(s, \rho)$ can be seen as a polynomial matrix in variable $s$ with coefficients over the field $\mathrm{K}(\rho)$ of multivariate rational functions: $S(s, \rho) \in \mathrm{K}(\rho)[s]^{\left(n+p+m_{u}\right) \times\left(n+m_{u}\right)}$. Alternatively, $S(s, \rho)$ can be interpreted as $S(s, \rho) \in$ $\mathrm{K}(\rho, s)^{\left(n+p+m_{u}\right) \times\left(n+m_{u}\right)}$, thus having elements in the field of rational functions in the variable $s$ and components of $\rho$. The latter interpretation ignores the polynomial structure of $S(s, \rho)$. Symbolic methods to compute nullspace bases of multivariate rational matrices are implemented in several popular computational platforms as MATLAB, Mapple, or Mathematica. The underlying algorithms of the function null of MATLAB, NullSpace of Maple, and NullSpace of Mathematica are based on matrix reductions to echelon forms using Gaussian elimination techniques and do not exploit the matrix polynomial structure of $S(s, \rho)$ with respect to $s$. As consequence, the resulting basis is formed by the columns of a rational matrix. To arrive to a polynomial basis in variable $s$, appropriate normalizations must be performed to eliminate the denominators. Therefore, better suited is the function MinimalBasis of Maple, which is able to compute a minimal polynomial basis (Kailath, 1980) with respect to variable $s$ and thus can be used to build detectors of least dynamical order. In what follows, we will assume that we use a minimal polynomial basis $N_{l}(s, \rho)$.

Regarding the computation of minimal polynomial basis, recent advances in computational algorithms are reported in (Storjohann and Villard, 2005) and (Jeannerod and Villard, 2006) and rely on randomization techniques. These algorithms are able to compute the minimal left nullspace basis $Y_{l}(s, \rho)$ of the linear pencil $S(s, \rho)$ with a computational complexity of $O\left(\left(n+p+m_{u}\right)^{\omega}\right)$, where $2 \leq \omega<2.376$ is the so-called exponent for matrix multiplication over the field $\mathrm{K}(\rho)$.

The resulting minimal polynomial basis $N_{l}(s, \rho)$ consists of $p-r_{d}$ row vectors. We assume that the $i$-th vector has degree $\nu_{i}$ and the vectors are ordered such that $\nu_{i+1} \geq \nu_{i}$, 
for $i=1, \ldots, p-r_{d}-1$. The degrees $\nu_{i}$ of a minimal basis are called minimal indices. $N_{l}(s, \rho)$ can be easily transformed into a physically realizable proper rational basis (and thus into a candidate detector), by simply premultiplying it with a diagonal TFM $M^{-1}(s)$, where $M(s)$ has as its $i$-th diagonal element an arbitrary polynomial $d_{i}(s)$ of degree $\nu_{i}$. The resulting TFM $M^{-1}(s) N_{l}(s, \rho)$ is proper and its poles are the roots of polynomials $d_{i}(s)$. The total order of the resulting realization is $\sum_{i=1}^{p-r_{d}} \nu_{i}$. Candidate detectors of lower orders can be obtained by selecting linear combinations of basis vectors up to a given order $\kappa$, where $\min \nu_{i} \leq \kappa \leq \max \nu_{i}$. This corresponds to replace $N_{l}(s, \rho)$ by $H N_{l}(s, \rho)$, where $H$ is a $q \times\left(p-r_{d}\right)$ full row rank constant matrix. As a candidate scalar output detector of order at most $\kappa, H$ can be chosen a vector $h$ of the form $h=\left[h_{1} h_{2}, \ldots, h_{k}, 0, \ldots, 0\right]$, where $k$ is chosen to include all vectors up to degree $\kappa$.

The resulting filter $Q(s, \rho)=M^{-1}(s) H N_{l}(s, \rho)$ can be transformed into a state-space realization of the form

$$
Q(s, \rho)=\left[\begin{array}{l|l}
A_{Q} & B_{Q}(\rho) \\
\hline C_{Q} & D_{Q}(\rho)
\end{array}\right]
$$

where $A_{Q}$ and $C_{Q}$ are constant matrices, and only $B_{Q}(\rho)$ and $D_{Q}(\rho)$ depend on $\rho$. This realization can be obtained by inspection, by concatenating the realizations of each row of $Q(s, \rho)$. The resulting realization of the $i$-th row has a realization $\left(A_{Q, i}, B_{Q, i}(\rho), C_{Q, i}, D_{Q, i}(\rho)\right)$, where the constant pair $\left(A_{Q, i}, C_{Q, i}\right)$ can be chosen in an observability companion form (Kailath, 1980), while the elements of $B_{Q, i}(\rho)$ and $D_{Q, i}(\rho)$ can be read off from the series expansion of $i$-th row polynomial vector. The resulting $A_{Q}$ and $C_{Q}$ are block-diagonal matrices of appropriate sizes.

The realization of the TFM from faults to residual $R_{f}(s, \rho)$ can be explicitly obtained in the form

$$
R_{f}(s, \rho)=M^{-1}(s) H Y_{l}(s, \rho)\left[\begin{array}{c}
B_{f}(\rho) \\
D_{f}(\rho) \\
0
\end{array}\right]=\left[\begin{array}{c|c}
A_{Q} & \widetilde{B}_{f}(\rho) \\
\hline C_{Q} & \widetilde{D}_{f}(\rho)
\end{array}\right],
$$

thus sharing with the realization of $Q(s, \rho)$ in (15) the constant matrices $A_{Q}$ and $C_{Q}$.

\subsection{Computation of approximative detectors}

In the case when $\rho$ contains both non-measurable components $\rho_{1}$ as well as measurable ones $\rho_{2}$ we can use the computed $Q(s, \rho):=Q\left(s, \rho_{1}, \rho_{2}\right)$ to obtain a suitable approximation $\bar{Q}\left(s, \rho_{2}\right)$ depending only of $\rho_{2}$. The solution of the approximation problems (13) or (14) can be addressed by exploiting the form of the realization (15) by choosing $\bar{Q}\left(s, \rho_{2}\right)$ to share the constant matrices $A_{Q}$ and $C_{Q}$

$$
\bar{Q}\left(s, \rho_{2}\right)=\left[\begin{array}{c|c|}
A_{Q} & \bar{B}_{Q}\left(\rho_{2}\right) \\
\hline C_{Q} & \bar{D}_{Q}\left(\rho_{2}\right)
\end{array}\right]
$$

There are several possibilities to robustly fit $\bar{B}_{Q}\left(\rho_{2}\right)$ and $\bar{D}_{Q}\left(\rho_{2}\right)$ to $B_{Q}\left(\rho_{1}, \rho_{2}\right)$ and $D_{Q}\left(\rho_{1}, \rho_{2}\right)$, respectively. We mention a few of them in what follows:

Structure preserving fitting: This involves finding a value $\bar{\rho}_{1}$ of $\rho_{1}$ which produces the best approximation $\bar{B}_{Q}\left(\rho_{2}\right):=B_{Q}\left(\bar{\rho}_{1}, \rho_{2}\right)$, by solving

$$
\bar{\rho}_{1}=\arg \min _{z \in \Pi_{1}} \max _{\rho \in \Pi}\left\|B_{Q}\left(\rho_{1}, \rho_{2}\right)-B_{Q}\left(z, \rho_{2}\right)\right\|
$$

Similar computation is performed for $\bar{D}_{Q}\left(\rho_{2}\right)$.

Parameter fitting: We can assume for $\bar{B}_{Q}\left(\rho_{2}\right)$ a certain parametric form $\widetilde{B}_{Q}\left(\rho_{2}, \theta\right)$ (e.g., affine, polynomial, rational, etc.) and fit the free parameters $\theta$ by solving

$$
\bar{\theta}=\arg \min _{\theta} \max _{\rho \in \Pi}\left\|B_{Q}(\rho)-\widetilde{B}_{Q}\left(\rho_{2}, \theta\right)\right\|
$$

and define $\bar{B}_{Q}\left(\rho_{2}\right):=\widetilde{B}_{Q}\left(\rho_{2}, \bar{\theta}\right)$ (and similarly for $\left.\bar{D}_{Q}\left(\rho_{2}\right)\right)$.

Input-output fitting: The fitting is performed by globally minimizing a suitable worst-case system norm

$$
\max _{\rho \in \Pi}\left\|Q(s, \rho)-\bar{Q}\left(s, \rho_{2}\right)\right\|
$$

For $\bar{B}_{Q}\left(\rho_{2}\right)$ and $\bar{D}_{Q}\left(\rho_{2}\right)$ in (16) either a structure preserving form or a parametric form can be used.

Weighted input-output fitting: The fitting is performed by globally minimizing the worst-case weighted system norm

$$
\max _{\rho \in \Pi}\left\|\left(Q(s, \rho)-\bar{Q}\left(s, \rho_{2}\right)\right) G_{e}(s, \rho)\right\|
$$

For $\bar{B}_{Q}\left(\rho_{2}\right)$ and $\bar{D}_{Q}\left(\rho_{2}\right)$ in (16) either a structure preserving form or a parametric form can be used.

When using standard optimization tools to solve the above min-max parameter fitting problems, the evaluation of the above criteria involves performing a worstcase optimization-based search. Thus, function evaluations are potentially expensive, and therefore an alternative is to replace the semi-infinite optimization problems by computationally tractable finite dimensional optimization problems. A frequently used approximation method is to use instead the continuous domain $\Pi$ only a discrete set of points $\Pi_{N}=\left\{\rho^{(1)}, \rho^{(2)}, \ldots, \rho^{(N)}\right\}$, obtained, for example, by parameter griding. By using a sufficiently dense grid of points, it is expected to obtain a satisfactory approximation of the continuous-case worst-case. One advantage of the gridding based approach is the possibility to perform in parallel all function evaluations necessary for the determination of worst-case maximum.

\section{ILLUSTRATIVE EXAMPLE}

We consider the robust actuator fault detection example of Edelmayer and Bokor (2002). The additive fault system has a standard state space realization (1) with

$$
\begin{gathered}
A\left(\rho_{1}, \rho_{2}\right)=\left[\begin{array}{ccc}
-0.8 & 0 & 0 \\
0 & -0.5\left(1+\rho_{1}\right) & 0.6\left(1+\rho_{2}\right) \\
0 & -0.6\left(1+\rho_{2}\right) & -0.5\left(1+\rho_{1}\right)
\end{array}\right] \\
B_{u}=\left[\begin{array}{ll}
1 & 1 \\
1 & 0 \\
0 & 1
\end{array}\right], B_{d}=0, B_{f}=\left[\begin{array}{ll}
1 & 1 \\
1 & 0 \\
0 & 1
\end{array}\right], C=\left[\begin{array}{lll}
0 & 1 & 1 \\
1 & 1 & 0
\end{array}\right] \\
D_{u}=0, D_{d}=0, D_{f}=0 .
\end{gathered}
$$

In the expression of $A\left(\rho_{1}, \rho_{2}\right), \rho_{1} \in[-0.25,0.25]$ and $\rho_{2} \in$ $[-0.25,0.25]$ are uncertainties in the real and imaginary parts of the two complex conjugated eigenvalues $\lambda_{1,2}=$ $-0.5 \pm j 0.6$ of the nominal state matrix $A(0,0)$. The fault detector filter is aimed to provide robust fault detection of actuator faults in the presence of these parametric uncertainties.

For the robust synthesis we consider the two cases, when either all parameters are measurable or non measurable. 
For each case, we evaluated the step responses of the parameter dependent residual generation system (of the form (4)) from the faults and control inputs on a uniform grid $\Pi_{N}$ for both $\rho_{1}$ and $\rho_{2}$ in the range $[-0.25,0.25]$, with $N=5 \times 5$ values.

\subsection{Case 1: Both $\rho_{1}$ and $\rho_{2}$ measurable}

We computed a minimal polynomial left nullspace basis $N_{l}(s, \rho)$ using the approach described in the previous section employing the Maple function MinimalBasis. The resulting $N_{l}(s, \rho)$ and the corresponding $N_{f}(s, \rho):=$ $N_{l}(s, \rho)\left[\begin{array}{c}G_{f}(s, \rho) \\ 0\end{array}\right]$ are given in Appendix A. It is easy to check that the RFDP is solvable, because each column of $N_{f}(s, \rho)$ is nonzero for all values of $\rho_{1}$ and $\rho_{2}$ in their variation range. Moreover, the RFDP is even solvable by using a first order scalar output detector constructed from the first row of $N_{l}(s, \rho)$ (of degree one). If we choose $d(s)=s+10$ and $h=\left[\begin{array}{ll}1 & 0\end{array}\right]$, then the corresponding detector is $Q(s, \rho)=h N_{l}(s, \rho) / d(s)$ and leads to

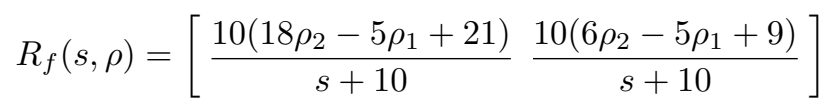

The corresponding DC-gain $R_{f}(0, \rho)$ is

$$
R_{f}(0, \rho)=\left[18 \rho_{2}-5 \rho_{1}+216 \rho_{2}-5 \rho_{1}+9\right]
$$

To normalize the smaller gain to unity, we take $Q(s, \rho)=$ $h N_{l}(s, \rho) / d(s) /\left(6 \rho_{2}-5 \rho_{1}+9\right)$ for which

$$
R_{f}(s, \rho)=\left[\begin{array}{ll}
\frac{10\left(18 \rho_{2}-5 \rho_{1}+21\right)}{(s+10)\left(6 \rho_{2}-5 \rho_{1}+9\right)} & \frac{10}{s+10}
\end{array}\right]
$$

and from (7) we get $\beta=1$. $Q(s, \rho)$ has a state-space realization $\left(A_{Q}, B_{Q}(\rho), C_{Q}, D_{Q}(\rho)\right)$ with $A_{Q}=-10, C_{Q}=$ 1 and $B_{Q}(\rho)$ and $D_{Q}(\rho)$ given in Appendix A.

In Figure 1 we present the results of parametric analysis of step responses. As it can be observed, a perfect decoupling is achieved for all parameter values from the control inputs. Thus, the detection of constant faults (e.g., abrupt changes) can be reliably performed in the presence of measurable parametric uncertainties.

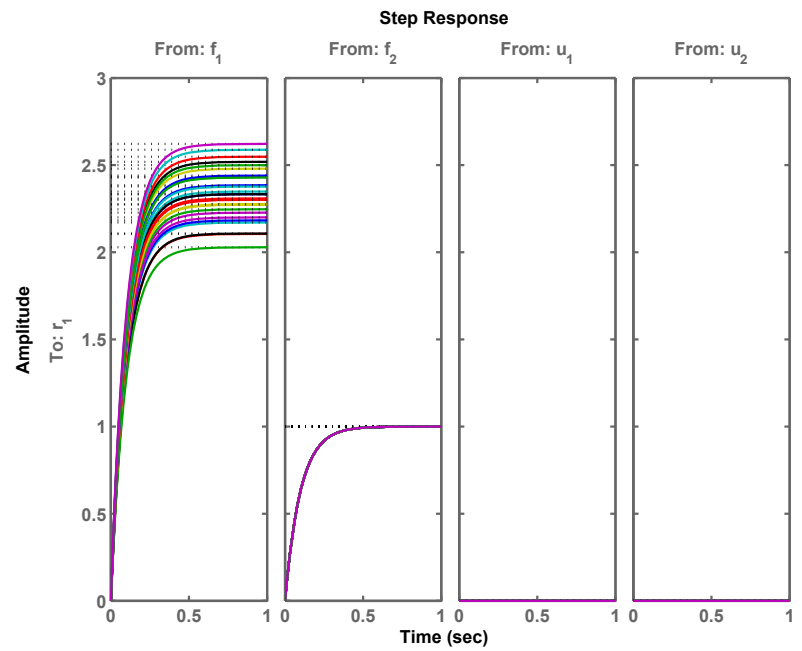

Fig. 1. Parametric step response analysis of the LPV detector robustness

\subsection{Case 2: Both $\rho_{1}$ and $\rho_{2}$ not measurable}

We determined the best approximations of $B_{Q}(\rho)$ and $D_{Q}(\rho)$ with constant matrices $\bar{B}_{Q}$ and $\bar{D}_{Q}$ over a grid of $N=25$ values using the parameter fitting approach. The resulting matrices are

$$
\begin{gathered}
\bar{B}_{Q}=\left[\begin{array}{llll}
24.4917 & -121.9081 & -23.4515 & -10.4306
\end{array}\right] \\
\bar{D}_{Q}=\left[\begin{array}{llll}
-3.2511 & 13.2508 & 0.0002 & 0.0001
\end{array}\right]
\end{gathered}
$$

We also determined the constant matrices $\bar{B}_{Q}$ and $\bar{D}_{Q}$ using the weighted input-output fitting of the $\mathcal{H}_{\infty}$-norm (17). The resulting matrices are

$$
\begin{gathered}
\bar{B}_{Q}=\left[\begin{array}{llll}
24.9718 & -122.073 & -23.5365 & -10.0792
\end{array}\right] \\
\bar{D}_{Q}=\left[\begin{array}{llll}
-3.2113 & 13.2406 & 0.0002 & 0.0001
\end{array}\right]
\end{gathered}
$$

The corresponding weighted $\mathcal{H}_{\infty}$-norm (17) is 0.6434 , while for the previous constant approximation obtained by parameter fitting this value was 0.8181 . For all cases, the global worst-case parameter combination corresponds to $\rho_{1}=\rho_{2}=-0.25$. The resulting parametric step responses for the approximation achieved using the weighted inputoutput fitting can be seen in Figure 2. As it can be observed, with an appropriate choice of the detection threshold, the detection and isolation of constant faults can be reliably performed in the presence of parametric uncertainties.

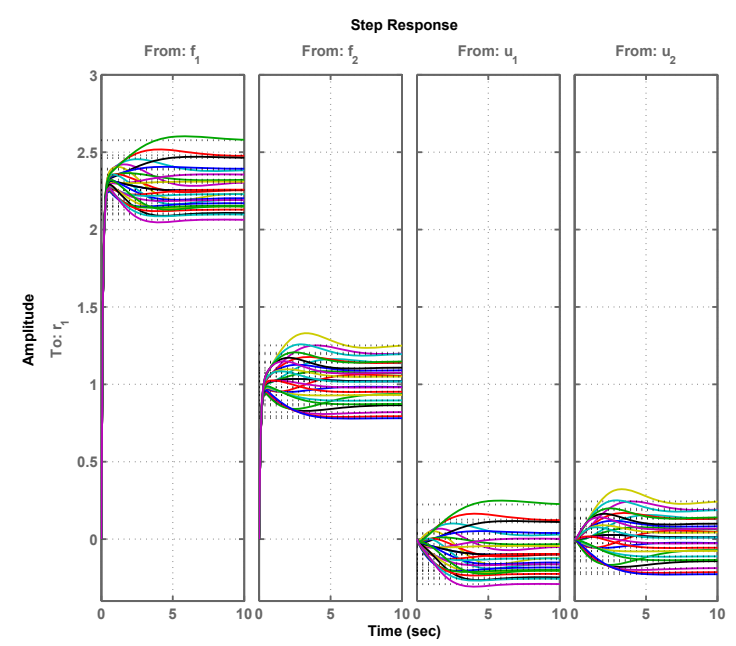

Fig. 2. Parametric step response analysis of constant weighted fitted detector robustness

\section{CONCLUSIONS}

We proposed a robust nullspace synthesis method of LPVscheduling based residual generators. Robustness against parametric uncertainties is provided by a worst-case search based tuning of the LPV-scheduling residual generator. All required symbolic and numerical computational tools are available in standard computational environments. The proposed synthesis method can be also employed for the parametric solution of the fault detection and isolation (FDI) problem using a bank of scalar detectors which provide a set of structured residuals. 
The computational performance can be enhanced in several ways. For example, instead of determining a full minimal nullspace basis, it is possible to determine only a part of this basis up to a specified degree and stop when the fault detectability condition is fulfilled. Adequate computational procedures for this purpose are described in (Storjohann and Villard, 2005). The symbolic computation of the nullspace basis can be even completely eliminated in the case when no measurable parameters exist. An appropriate choice of an initial constant detector can be determined using a nominal synthesis and this can serve for the parametrization of $Q(s)$ via a quadruple $\left(A_{Q}, B_{Q}, C_{Q}, D_{Q}\right)$, where $A_{Q}$ and $C_{Q}$ are fixed and $B_{Q}$ and $D_{Q}$ can be optimally determined.

Alternative techniques to address the (weighted) inputoutput fitting can be also considered, as for example solving parameter dependent linear matrix inequalities using convex relaxations techniques (Scherer, 2006) or using randomization methods to solve semi-infinite optimization problems (Tempo et al., 2004).

\section{REFERENCES}

Balas, G.J., Bokor, J., and Szabó, Z. (2003). Invariant subspaces for lpv systems and their application. IEEE Trans. Automat. Control, 48, 2065-2069.

Bokor, J. and Balas, G. (2004). Detection filter design for LPV systems a geometric approach. Automatica, 40, $511-518$.

Chen, J. and Patton, R.J. (1999). Robust Model-Based Fault Diagnosis for Dynamic Systems. Kluwer Academic Publishers, London.

Edelmayer, A. and Bokor, J. (2002). Optimal $\mathcal{H}_{\infty}$ scaling for sensitivity optimization of detection filters. Int. J. Robust Nonlinear Control, 12, 749-760.

Frisk, E. and Nyberg, M. (2001). A minimal polynomial basis solution to residual generation for fault diagnosis in linear systems. Automatica, 37, 1417-1424.

Jeannerod, C.P. and Villard, G. (2006). Asymptotically fast polynomial matrix algorithms for multivariable systems. Int. J. Control, 79, 1359-1367.

Kailath, T. (1980). Linear Systems. Prentice Hall, Englewood Cliffs, N.J.

Kinnaert, M., Hanus, R., and Arte, P. (1995). Fault detection and isolation for unstable systems. IEEE Trans. Automat. Control, 40, 740-742.
Nyberg, M. (2002). Criterions for detectability and strong detectability of faults in linear systems. Int. J. Control, $75,490-501$.

Pfifer, H. and Hecker, S. (2008). Generation of optimal linear parametric models for LFT-based robust stability analysis and control design. Proc. IEEE CDC'09, Cancun, Mexico.

Scherer, C. (2006). LMI relaxations in robust control. European Journal of Control, 12, 3-29.

Storjohann, A. and Villard, G. (2005). Computing the rank and a small nullspace basis of a polynomial matrix. Proc. of 2005 International Symposium on Symbolic and Algebraic Computation, Beijing, China, 309-316.

Tempo, R., Calafiore, G., and Dabbene, F. (2004). Algorithms for Analysis and Control of Uncertain Systems. Springer-Verlag, New York.

Varga, A. (2003). On computing least order fault detectors using rational nullspace bases. Proc. of IFAC Symp. SAFEPROCESS'2003, Washington D.C.

Varga, A. (2007). On designing least order residual generators for fault detection and isolation. Proc. 16th Internat. Conf. on Control Systems and Computer Science, Bucharest, Romania, 323-330.

Varga, A. (2008a). Linear FDI-Techniques and Software Tools. FAult Detection Toolbox V0.8 - Technical Documentation IB 515-08-18, German Aerospace Center (DLR), Institute of Robotics and Mechatronics.

Varga, A. (2008b). On computing nullspace bases - a fault detection perspective. Proc. IFAC 2008 World Congress, Seoul, Korea., 6295-6300.

Varga, A., Looye, G., Moormann, D., and Grübel, G. (1998). Automated generation of LFT-based parametric uncertainty descriptions from generic aircraft models. Mathematical and Computer Modelling of Dynamical Systems, 4, 249-274.

Vinnicombe, G. (1993). Frequency domain uncertainty and the graph topology. IEEE Trans. Automat. Control, 38, 1371-1383.

Wonham, W.M. (1979). Linear Multivariable Control: a Geometric Approach. Springer Verlag, New York.

Zhou, K., Doyle, J.C., and Glover, K. (1996). Robust and Optimal Control. Prentice Hall.

Appendix A.

$$
\begin{array}{r}
N_{l}(s, \rho)=\left[\begin{array}{cc}
-\left(50 \rho_{1}+60 \rho_{2}+30\right) s-25 \rho_{1}^{2}-36 \rho_{2}^{2}-10 \rho_{1}-120 \rho_{2}-69 & 120\left(\rho_{2}+1\right) s+96 \rho_{2}+96 \\
100 s^{2}+100\left(\rho_{1}+1\right) s+25 \rho_{1}^{2}+36 \rho_{2}^{2}+50 \rho_{1}+72 \rho_{2}+61 & 0 \\
50 \rho_{1}-180 \rho_{2}-210 & 50 \rho_{1}-60 \rho_{2}-90 \\
-100 s+60 \rho_{2}-50 \rho_{1}+10 & -100 s-50 \rho_{1}-60 \rho_{2}-110
\end{array}\right] \\
N_{f}(s, \rho)=\left[\begin{array}{ccc}
180 \rho_{2}-50 \rho_{1}+210 & 60 \rho_{2}-50 \rho_{1}+90 \\
100 s+50 \rho_{1}-60 \rho_{2}-10 & 100 s+50 \rho_{1}+60 \rho_{2}+110
\end{array}\right] \\
B_{Q}(\rho)=\left[\begin{array}{rrr}
-\frac{25 \rho_{1}^{2}-490 \rho_{1}+36 \rho_{2}^{2}-480 \rho_{2}-231}{6 \rho_{2}-5 \rho_{1}+9} & -\frac{1104\left(\rho_{2}+1\right)}{6 \rho_{2}-5 \rho_{1}+9} & \frac{10\left(5 \rho_{1}-18 \rho_{2}-21\right)}{6 \rho_{2}-5 \rho_{1}+9}
\end{array}\right] \\
D_{Q}(\rho)=\left[\begin{array}{rrr}
-\frac{10\left(5 \rho_{1}+6 \rho_{2}-3\right)}{6 \rho_{2}-5 \rho_{1}+9} & \frac{120\left(\rho_{2}+1\right)}{6 \rho_{2}-5 \rho_{1}+9} & 0
\end{array}\right]
\end{array}
$$

